\title{
How We Treat HER2-Positive Metastatic Breast Cancer
}

\author{
Ashok S. Komaranchath ${ }^{1}$ Aju Mathew ${ }^{2,3}$ \\ ${ }^{1}$ Department of Oncology, Aster Medcity, Kochi, Kerala, India \\ 2Department of Oncology, MOSC Medical College, Kolenchery, \\ Kerala, India \\ ${ }^{3}$ Department of Internal Medicine, Markey Cancer Center, \\ University of Kentucky, Lexington, Kentucky, United States \\ Ind J Med Paediatr Oncol 2021;42:370-375.
}

\section{Introduction}

Overexpression of the human epidermal growth factor receptor-2 (HER2), or amplification of the HER2 gene, is seen in approximately 15 to $25 \%$ of all breast cancer patients. ${ }^{1}$ Real-world data from hospital-based cohorts estimated a prevalence of $23 \%{ }^{2}$ Far more patients in India present with advanced disease when compared with high-income countries. In this narrative review, we describe the current landscape of HER2 targeting. We focus on how we treat HER2-positive metastatic breast cancer (MBC).

\section{What are the Therapeutic Options to Target HER2 Receptor?}

The first drug targeting the HER2 receptor, trastuzumab, was approved for use in MBC in September 1998. In March 2007, lapatinib, a novel dual tyrosine kinase inhibitor which inhibited both HER2 and EGFR - Epidermal Growth Factor Receptor was approved. In June 2012, pertuzumab, the second monoclonal antibody for HER2-positive MBC was approved based on the CLEOPATRA trial. ${ }^{3}$ It bound to the HER2 receptor at a different epitope, thereby preventing heterodimerization of HER2 with HER3 receptors, as compared with trastuzumab which inhibits the homodimerization of two HER2 receptors. In February 2013, a novel antibody-drug conjugate, ado-trastuzumab emtansine (T-DM1), was approved for use in MBC based on the EMILIA trial. Recently, three new drugs were approved for use in MBC: trastuzumab deruxtecan (T-DXd) in December 2019, neratinib in February 2020, and tucatinib in April 2020. Of note, there is no evidence to use HER2-targeted therapy in patients with HER2-negative disease, even if HER2 test by immunohistochemistry is scored at 2+ but Fluorescent In Situ Hybridization test is negative.

DOI https://doi.org/ $10.1055 / \mathrm{s}-0041-1731861$ ISSN 0971-5851

\begin{abstract}
Address for correspondence Aju Mathew, MBBS, MD, Dip AB, MPhil, FACP. Department of Oncology, MOSC Medical College, Kolenchery, Kerala, India (e-mail: cancerkerala@gmail.com).
\end{abstract}

\section{How We Treat HER2-Positive De Novo Metastatic Breast Cancer?}

\section{First-Line Setting}

For a patient with de novo HER2-positive MBC, we offer the combination of trastuzumab, pertuzumab, and docetaxel based on the CLEOPATRA Trial. ${ }^{3}$ Addition of pertuzumab to trastuzumab and docetaxel showed a significant improvement in both PFS - Progression Free Survival (18.7 vs. 12.4 months, $p<0.001$ and OS - overall survival (56.5 vs. 40.8 months, $p<0.001$ ) as compared with the use of placebo. Risk for cardiac toxicity (left ventricular dysfunction) was lower in the pertuzumab arm (6.6 vs. $8.6 \%$ ). If patient has responded to the treatment regimen, we discontinue docetaxel after six to eight cycles. Trastuzumab and pertuzumab are continued until progression or intolerability. There is no evidence with a strategy to just use pertuzumab for a few doses. Financial considerations must be factored in while taking a decision on adding pertuzumab.

For patients with hormone receptor-positive and HER2-positive disease (often referred as the triple positive disease), antihormonal therapy is often added after the chemotherapy is concluded (along with maintenance trastuzumab and pertuzumab). A high level of ER - Estrogen receptor expression has several implications on the biology of the tumor, as there is abundant evidence of numerous cross-talk between the ER and HER2 signaling pathways. A subset analysis from a clinical trial showed that the efficacy of trastuzumab was lower in patients with triple positive disease treated with trastuzumab and chemotherapy when compared with patients with hormone receptor-negative disease. ${ }^{4} \mathrm{As}$ a result, the prognosis for patients with triple positive disease may be inferior compared with those patients with hormone receptor-negative and HER2-positive disease. ${ }^{5}$

\footnotetext{
(C) 2021. Indian Society of Medical and Paediatric Oncology.

This is an open access article published by Thieme under the terms of the Creative Commons Attribution-NonDerivative-NonCommercial-License, permitting copying and reproduction so long as the original work is given appropriate credit. Contents may not be used for commercial purposes, or adapted, remixed, transformed or built upon. (https://creativecommons.org/licenses/by-nc-nd/4.0/).

Thieme Medical and Scientific Publishers Private Ltd. A-12, Second Floor, Sector -2, NOIDA -201301, India
} 
For patients who are frail, we do offer a chemotherapy-free regimen comprising of just trastuzumab and pertuzumab. There is no evidence for such a strategy in the metastatic setting. However, data from the neoadjuvant NeoSphere trial showed that trastuzumab and pertuzumab resulted in producing pathologic complete response in around 17\% of patients with early-stage or locally advanced breast cancer. ${ }^{6}$ We extrapolate these findings to suggest that in a situation where chemotherapy is absolutely or relatively contra-indicated, targeted therapy with these monoclonal antibodies could be considered. If such patients have hormone receptor positive disease, we add anti-hormonal therapy concurrently with anti-HER2 therapy.

We practice alternative approaches within a resource-constrained setting. For instance, we use pertuzumab for a limited duration along with docetaxel and trastuzumab with an aim to increase the response rate, unlike the CLEOPATRA regimen where the drug is used indefinitely until intolerability or progression ensues. Most often, we are unable to add pertuzumab due to the significant cost burden to the patient. In situations where patients cannot afford trastuzumab, we recommend any tolerable chemotherapy option, preferably as monotherapy. Some patients may consider anti-HER2 targeting using lapatinib (generic versions are cheaper than biosimilar trastuzumab). For patients who have large tumor burden in visceral organs, we may often use doublet chemotherapy with a goal to reverse the impending visceral crisis, especially if we are unable to use a HER2 targeting agent. Doublet chemotherapy produces more tumor response than monotherapy but may not always provide an overall survival benefit.

\section{Subsequent Lines of Therapy}

Geyer et al randomized 324 patients with advanced HER2-positive breast cancer who had progressed following treatment that included an anthracycline, a taxane, and trastuzumab to either capecitabine alone $\left(2,500 \mathrm{mg} / \mathrm{m}^{2} /\right.$ day D1-D14 Q21d) or capecitabine $\left(2,000 \mathrm{mg} / \mathrm{m}^{2} /\right.$ day D1-D14 Q 21d) plus lapatinib $\left(1,250 \mathrm{mg} /\right.$ day continuously). ${ }^{7}$ The primary endpoint, time to progression (TTP), was 8.4 months in the lapatinib arm versus 4.4 months in the capecitabine only $\operatorname{arm}$ (hazard ratio $=0.47 ; p<0.001$ ). There were no symptomatic cardiac events with lapatinib. Diarrhea and rash were more commonly seen in patients receiving lapatinib and capecitabine compared with capecitabine alone. With the publication of this trial, lapatinib plus capecitabine became the best second-line treatment option.

The results of the EMILIA trial, a phase-III trial with 991 patients randomized to receive either trastuzumab emtansine (T-DM1) or lapatinib plus capecitabine created a new standard of care for second-line setting. ${ }^{8}$ PFS (9.6 vs. 6.4 months, $p<0.001$ ) and OS (30.9 vs. 25.1 months, $p<0.001$ ) were significantly prolonged with the use of T-DM1. It was also better tolerated than lapatinib and capecitabine. But for the vast majority of patients in India, T-DM1 is unaffordable.

Another option for patients who progress on trastuzumab is to continue trastuzumab and add pertuzumab along with chemotherapy, extrapolating from the positive results of the CLEOPATRA trial. ${ }^{3}$ Continuing trastuzumab beyond the progression and changing the chemotherapy backbone is a viable alternative approach. In the German Breast group/BIG 03-05 study, patients with disease progression while receiving trastuzumab were randomized to capecitabine plus continuation of trastuzumab versus capecitabine alone. A significant benefit in median TTP of 8.2 versus 5.6 months was noted $(p=0.033) .{ }^{9}$ The EGF104900 study investigated the addition of lapatinib to trastuzumab versus lapatinib alone. ${ }^{10}$ The results in this heavily pretreated HER2-positive MBC cohort revealed a significant PFS and OS benefit with an absolute improvement in overall survival of $10 \%$ at 6 months and $15 \%$ at 12 months. There was a significant 4.5-month OS advantage with dual HER2 blockade.

Neratinib (a novel small molecule TKI with anti-HER2 activity) plus capecitabine is another alternative for patients with HER2-positive MBC who have received at least two prior lines of HER2-directed treatment. The NALA trial, which compared neratinib plus capecitabine with lapatinib plus capecitabine, found significant PFS benefit (hazard ratio $[\mathrm{HR}]=0.76 ; 95 \%$ confidence interval $[\mathrm{CI}]: 0.63-0.93 ; p=$ 0.0059) for the newer drug. ${ }^{11}$ Currently, neratinib is not available in India outside of compassionate use.

Likewise, two new promising anti-HER2 drugs, tucatinib and trastuzumab deruxtecan, which were recently approved in the United States, are not available in India.

The HER2CLIMB trial was a phase II trial conducted in heavily pretreated HER2 positive MBC patients who had progressed on trastuzumab, pertuzumab, and T-DM1. Either tucatinib or placebo were added to trastuzumab plus capecitabine and continued till progression. Primary endpoint, PFS, at 1 year was 33.1 versus $12.3 \%(p<0.001)$ and median PFS was 7.8 versus 5.6 months. Overall survival was also better at 2 years with $44.9 \%$ in the tucatinib arm as compared with $26.6 \%$ in the placebo arm. ${ }^{12}$ Tucatinib was also noted to be very effective in patients with brain metastases. PFS at 1 year in this subset of patients was $24.9 \%$ as compared with $0 \%$ in the placebo arm. The risk of disease progression or death was $52 \%$ lower in the tucatinib arm ( $\mathrm{HR}=0.48 ; 95 \%$ CI: $0.34-0.69 ; p<0.001)$. This finding was particularly significant as brain metastases is an important cause of morbidity and mortality in HER2-positive MBC.

Trastuzumab deruxtecan is a new antibody-drug conjugate which uses a proprietary tetrapeptide-based linker which combines the anti-HER2 antibody with a cytotoxic payload, a potent topoisomerase-I inhibitor. This new drug has a higher drug-to-antibody ratio than T-DM1 and the linker is stable in plasma. It is selectively cleaved at the tumor site by cathepsins which are upregulated specifically in tumor cells. The single-arm phase-II DESTINY-Breast01 trial found an overall response rate of $60.3 \%$ with a $4.3 \%$ complete response rate and $56 \%$ partial response rate. ${ }^{13}$ No comparative trials with this molecule have been published till date. Incidence of interstitial lung disease (ILD) was observed in $2.6 \%$ of the study population.

For patients who have hormone receptor positive disease, there is an option to change the antiestrogen therapy with disease progression. There are no trials that will guide the 
decision-making in such an instance. Repeat biopsy (tissue or liquid) to look for any evidence of hormone resistance mutations may be a clinically beneficial strategy.

\section{How We Treat HER2-Positive Metastatic Breast Cancer Progressed after Adjuvant Therapy?}

These days, most HER2-positive nonmetastatic patients receive at least one HER2 directed agent as part of adjuvant therapy. Although most doctors in high-income countries use adjuvant trastuzumab for 1 year, its incremental benefit over a shorter course is probably marginal. The results from the PERSEPHONE trial showed that 6 months of adjuvant trastuzumab could be noninferior to 1 year of therapy in early stage breast cancer. ${ }^{14}$ In India, it is not uncommon to see even shorter duration of trastuzumab. A 9-week course of trastuzumab based on the FinHer trial is often used in resource limited settings. ${ }^{15}$ In some settings, patients who cannot access trastuzumab due to affordability or lack of financial assistance programs are treated with just chemotherapy.

The key question in the setting of progression following adjuvant therapy is to determine if the patient has trastuzumab resistance. In the BOLERO-3 trial, trastuzumab resistance was defined as recurrence during or within 12 months of completing adjuvant treatment or progressive disease within 4 weeks of treatment in the metastatic setting. ${ }^{16}$ However, this definition is widely debated and there is a lack of consensus regarding the treatment-free interval (TFI) necessary to define resistance. The U.S. Food and Drug Administration (FDA) uses a cut-off of 6 months of TFI to determine trastuzumab resistance. The European $\mathrm{ABC}-4$ guidelines suggest a TFI of 12 months to define resistance to trastuzumab.

\section{First-Line Setting}

TFI $\geq 6$ months: Patients with a treatment-free duration of 6 months or more following adjuvant trastuzumab would likely benefit from dual HER2 blockade (trastuzumab plus pertuzumab) plus a taxane, based on the CLEOPATRA Trial. ${ }^{9}$ It would still be a good choice if disease progression occurs after 6 months of stopping adjuvant T-DM1 (a new option for adjuvant treatment if neoadjuvant anti-HER2 treatment is given with trastuzumab and a pathologic complete response [pCR] is not achieved). However, if disease progression occurs after 6 months of stopping adjuvant pertuzumab (a new option for adjuvant treatment if neoadjuvant anti-HER2 treatment is given with trastuzumab and a PCR is not achieved), we suggest either a rechallenge with CLEOPATRA regimen or switching to T-DM1 based on EMILIA trial regimen.

TFI $<6$ months: Patients with a treatment-free duration of less than 6 months following adjuvant trastuzumab would likely benefit from trastuzumab emtansine (T-DM1). This is based on the phase-III trials, EMILIA, and TH3RESA which led to the FDA approval of T-DM1., ${ }^{8,17}$ The former trial compared T-DM1 with the combination of lapatinib plus capecitabine and the latter compared it with treatment of physician's choice of which most patients received trastuzumab plus chemotherapy. Both trials showed significant PFS and OS benefit of T-DM1 over the comparator. However, in the subset of patients who may have received T-DM1 as adjuvant therapy as mentioned above, other options involving cytotoxic chemotherapy may have to be sought. Newer options such as trastuzumab deruxtecan or tucatinib (in combination with capecitabine and trastuzumab) are unavailable in India as of now.

\section{Subsequent Lines of Therapy}

The best option for patients who have received prior trastuzumab (with or without pertuzumab) would be to use T-DM1. Beyond that, various cytotoxic chemotherapy options with trastuzumab or lapatinib may need to be used. Both trastuzumab deruxtecan (DESTINY-Breast01 trial) ${ }^{13}$ and tucatinib (HER2CLIMB trial) ${ }^{12}$ have shown benefit in heavily pretreated HER2-positive advanced breast cancer patients, all of whom had prior exposure to T-DM1. The NALA trial for neratinib had only approximately $50 \%$ of patients who had prior exposure to T-DM1. ${ }^{11}$ These drugs are not easily accessible in India.

"Triple-positive" breast cancer: There has been little consensus on the addition of hormonal agents in this subset of patients. A recent trial called the ALTERNATIVE trial, randomized 355 postmenopausal patients with prior treatment with both trastuzumab and endocrine therapy either in the adjuvant or metastatic setting, to three arms, namely, lapatinib plus trastuzumab plus AI - Aromatase Inhibitor versus lapatinib plus AI versus trastuzumab plus AI. ${ }^{18}$ Any one of the three AIs (letrozole, anastrozole, and exemestane) could be used. All three arms did not receive chemotherapy. The primary endpoint was PFS. The updated results showed that dual HER2 blockade along with an AI had significant PFS benefit over the combination of AI with Trastuzumab (11 vs. 5.6 months; $\mathrm{HR}=0.62$ [95\% CI: 0.45-0.88]; $p=0.0063$ ).

Another breakthrough in the treatment of hormone-positive advanced breast cancer has been the discovery of CDK4/6 inhibitors. However, data regarding the use in so-called triple-positive advanced breast cancers are scanty. A recent three-arm, phase-II trial called monarcHER looked into the possibility of incorporating CDK4/6 inhibitors with HER2-directed therapy in patients with hormone-positive, HER2-positive advanced breast cancer who have already undergone treatment with at least two anti-HER2 agents. ${ }^{19}$ Patients were randomized to receive either abemaciclib plus trastuzumab plus fulvestrant (group A) or abemaciclib plus trastuzumab (group B) or the standard-of-care chemotherapy (as per physician's choice) plus trastuzumab (group C). The most common chemotherapeutic agents used were vinorelbine, capecitabine, eribulin, or gemcitabine. The primary endpoint, median PFS, was 8.3 months (group A) versus 5.7 months (group C); HR $=0.67, p=0.051$. Of note, all patients in the study had prior exposure to trastuzumab and T-DM1, with approximately $54 \%$ exposed to pertuzumab as well. There was no difference in PFS between groups B and C. The overall survival data were not mature. 
These two trials open a possibility to use a chemotherapy-free regimen in such "triple-positive" breast cancer patients, even in heavily pretreated settings.

\section{What Are the Pertinent Side Effects of HER2-Targeted Therapies?}

Targeted therapy generally has fewer side effects when compared with cytotoxic chemotherapy. However, there are some unique side effects associated with HER2-targeted therapies. Cardiac toxicity is the major concern with trastuzumab. The incidence rate in adjuvant trials are less than $2 \%$, majority is asymptomatic. Trastuzumab cardiotoxicity is reversible if recognized early and intervened appropriately. A drop in left ventricular ejection fraction (LVEF) warrants withholding trastuzumab $(\geq 16 \%$ absolute reduction in left ventricular ejection fraction from baseline values or if the ejection fraction is already lower than the lower limit of normal, $a \geq 10 \%$ absolute drop in ejection fraction). But the drug can be reintroduced cautiously if the heart function recovers. Further drop in LV dysfunction, in our opinion, necessitates permanent discontinuation of trastuzumab. The safety of reintroducing trastuzumab in such a setting has not been studied. Although there are no contraindications for the use of trastuzumab, we recommend against its use in patients with heart failure or in patients with persistent drop in heart function, even after withholding the drug for 4 weeks. In the adjuvant setting, all patients receiving trastuzumab should have their LVEF recorded at baseline and once every 3 months while on treatment. However, we generally avoid such stringent monitoring of LVEF when trastuzumab is used in a metastatic setting. The risk-benefit balance for detecting asymptomatic drop in heart function is different in a curative setting versus a metastatic setting. Therefore, we primarily rely on clinical assessment for heart failure.

The side-effect profile and incidences of most adverse events with pertuzumab were like trastuzumab. The incidence of any LV dysfunction was slightly lesser in the taxane plus pertuzumab plus trastuzumab arm (6.6\%) than in the taxane plus trastuzumab arm (8.6\%) in the CLEOPATRA trial. ${ }^{9}$

Lapatinib, an oral tyrosine kinase inhibitor, has a higher incidence of diarrhea and skin toxicities especially when combined with capecitabine. There was also around 10\% incidence of low-grade musculoskeletal events. Around 40 to $50 \%$ of patients developed some amount of hepatotoxicity but only $4 \%$ were of grade- 3 and $<1 \%$ with grade- 4 toxicity.

T-DM1 has a higher incidence of thrombocytopenia (28\% overall and $12.9 \%$ grade $3 / 4$ in EMILIA trial). There was also a $22 \%$ incidence of deranged liver enzymes with around $4 \%$, being grade $3 / 4$. In view of this, monitoring LFT - liver function test monthly for the first 3 months and then once in 3 months is recommended.

Trastuzumab deruxtecan has a side-effect profile like T-DM1. Neutropenia was slightly more common than thrombocytopenia and was the most common grade $3 / 4$ adverse event. However, interstitial lung disease was diagnosed in $9 \%$ of the study population in the DESTINY-Breast01 trial and led to death in $2.6 \%$ of patients.
Neratinib has a high incidence for grade 3/4 diarrhea, as high as $40 \%$ in the ExteNET trial. ${ }^{20}$ Therefore, intensive loperamide prophylaxis should be used for all patients simultaneous with the start of therapy. Patients also had vomiting, rash, fatigue, and stomatitis but were very rarely severe. Hepatotoxicity was also concerning with around 9\% reporting elevated liver enzymes.

Diarrhea is the most common side effect with the use of tucatinib with an incidence of $81 \%$ (grade $3 / 4,12.5 \%$ ). Vomiting, rash, and hepatotoxicity (42\% overall and $9 \%$ grade $3 / 4$ ) were also reported with the drug.

\section{What Is New?}

\section{Drug Resistance Mechanisms}

Resistance to trastuzumab may be intrinsic or acquired. Intrinsic resistance develops before application of therapy and is usually related to a fault in the target receptor (e.g., a truncated HER2 receptor lacking the extracellular binding domain for trastuzumab or epitope masking by MUC4 or CD44/hyaluronan polymeric complex) or alterations in the PI3K/AKT/mTOR downstream signaling pathway. Acquired resistance usually involves target signaling and the target receptor. This includes upregulation of the signaling pathways downstream to HER2 or activation of an alternative signaling pathway (e.g., insulin-like growth factor-1 receptor, c-MET receptor, reduced levels of p27 expression, CXCR4 overexpression, EGFR - Epidermal Growth Factor Receptor homodimers, and EGFR/HER3 heterodimers). Receptor "cross-talk" between ER and HER2 have been studied extensively and the interactions between these have been found to be bidirectional and complex. Several trials (TanDEM, eLEcTRA, and EGF30008) have demonstrated significant PFS benefit in the metastatic setting for inhibiting both ER and HER2 pathways rather than ER inhibition alone. ${ }^{21-23}$ However, an OS benefit has not been demonstrated in these trials.

Finally, it has been discovered that certain genetic polymorphisms (e.g., those producing the phenotype expression of valine (V) or phenylalanine (F) at amino acid 158 on the FcrRIIIa) may influence the affinity of immunoglobulin (Ig)G1 to the Fcr receptor thereby reducing the ability to generate an adequate antibody-dependent cellular cytotoxicity (ADCC) from the effector cells. This was found to significantly reduce both response rates and PFS in the metastatic setting in patients treated with trastuzumab. ${ }^{24}$

\section{Immunotherapy}

In the NeoSphere trial, the expression of PD-1 receptor and its ligand PDL-1 was associated with a lower rate of pathological complete response. ${ }^{6}$ A genomic analysis study revealed that certain immune function genes are linked to clinical outcome in patients receiving trastuzumab. ${ }^{25}$ Several clinical trials combining HER2-directed therapy with immune checkpoint inhibitors are now underway.

Studies have also shown that HER2-positive breast cancer tends to have higher percentage of PDL-1 expression and hence was associated with high TILs and better overall survival. ${ }^{26}$ Lymphocyte-predominant breast cancer (LPBC) is 
defined as tumors with more than 50 to $60 \%$ of TILs, and have a better prognosis even if they belong to a less-favorable subtype such as HER2+ or TNBC. ${ }^{27}$ LPBC is seen in $20 \%$ of TNBC, $16 \%$ of HER2 subtype, and only $6 \%$ of hormone-positive subtypes. ${ }^{28}$ However, trials so far have not shown definitive benefits. Two phase-II trials, PANACEA (pembrolizumab + trastuzumab) and KATE2 (atezolizumab + T-DM1) showed no significant benefit in pretreated HER2-positive patients. ${ }^{29,30}$ As with most instances of immunotherapy, an ideal biomarker for patient selection seems to be the key to greater response.

\section{New Therapeutic Options}

Newly engineered HER2-directed therapies are being developed. Margetuximab is a chimeric IgG1antibody with a Fab fragment identical to trastuzumab but an Fc fragment modified to provide increased affinity for high-affinity and low-affinity isoforms of FcrR-IIIA and decreased affinity toward FcyR-IIIB in immune cells. This is postulated to improve cell kill through Fc-dependent methods like ADCC.

T-cell bispecific antibodies are novel molecules which can bind to both the CD3 chain of T-cell receptor and to tumor-specific antigens. A novel molecule MCLA-128 has been developed which targets both HER2 and HER3 receptors with enhancement in ADCC. It was designed to overcome HER3-mediated resistance to HER2-directed therapy. It was also found to have improved efficacy targeting tumor cells with low amounts of HER2 expression.

Complex immunotherapy combinations are another area of active interest. The ongoing AVIATOR TBCRC045 trial is comparing utomilumab (a novel CD137/4-1BB agonist) plus avelumab versus a combination of trastuzumab, vinorelbine, and avelumab in HER2-positive patients who have progressed after both trastuzumab and pertuzumab. ${ }^{31}$ It is one of the earliest trials to test the use of a costimulatory agonist with a PD-L1 inhibitor.

\section{New Routes of Administration}

FDA recently approved the subcutaneous formulation of trastuzumab called Herceptin Hylecta which combines trastuzumab with hyaluronidase based on the neoadjuvant HannaH trial which showed similar pCR rates to intravenous trastuzumab. ${ }^{32}$ There is no loading dose. The standard dose is 600 - $\mathrm{mg}$ trastuzumab and 10,000 units of hyaluronidase $(600 / 10,000)$ subcutaneously over 2 to 5 minutes once every 3 weeks.

Intrathecal trastuzumab has also been of interest, especially in patients with leptomeningeal disease which is not an uncommon site of metastasis in HER2-positive patients. ${ }^{33}$ In the rare situation where there is leptomeningeal disease in the presence of excellent extra-cranial disease control or absence of extracranial disease, as well as brain parenchymal disease, we have used intrathecal trastuzumab. There are no prospective trials that test such a therapeutic strategy.

\section{Conclusion}

From a relatively hard-to-treat and poor prognosis subtype of breast cancer, the past two decades have seen a quantum leap in the understanding, approach, management, and survival in HER2-positive disease. With the newer targeted agents currently available, it is not unreasonable to expect similar survival in HER2-positive MBC as compared with HER2-negative MBC. By unravelling newer mechanisms of interaction and resistance to the HER2 signaling pathway, we may be able to improve quality of life and survival. However, the cost of care is a major hurdle. In a resource-poor country, such as ours, access to first-line trastuzumab, even with cheaper generic options, is unaffordable for many, let alone the exorbitant costs of newer targeted therapies. Generic formulations of trastuzumab, including an FDA-approved biosimilar, as well as multiple generic formulations of lapatinib, have greatly improved cost effectiveness and compliance in India. It is now possible to obtain a 440-mg vial of trastuzumab for around Rs. 20,000 (U.S. \$250-300) and a month of lapatinib for around Rs. 10,000 (U.S. \$150). Hopefully, newer advances and incremental reduction of costs may make these highly effective molecules accessible to all.

\section{Conflict of Interest}

None declared.

\section{References}

1 Wolff AC, Hammond MEH, Hicks DG, et al. American Society of Clinical Oncology; College of American Pathologists. Recommendations for human epidermal growth factor receptor 2 testing in breast cancer: American Society of Clinical Oncology/College of American Pathologists clinical practice guideline update. J Clin Oncol 2013;31(31):3997-4013

2 Lux MP, Nabieva N, Hartkopf AD, et al. Therapy landscape in patients with metastatic HER2-positive breast cancer: data from the PRAEGNANT real-world breast cancer registry. Cancers (Basel) 2018;11(1):10

3 Swain SM, Baselga J, Kim SB, et al. CLEOPATRA Study Group. Pertuzumab, trastuzumab, and docetaxel in HER2-positive metastatic breast cancer. N Engl J Med 2015;372(8):724-734

4 Vici P, Pizzuti L, Sperduti I, et al. "Triple positive" early breast cancer: an observational multicenter retrospective analysis of outcome. Oncotarget 2016;7(14):17932-17944

5 Iancu G, Vasile D, Iancu RC, DaviȚoiu DV. "Triple positive" breast cancer - a novel category? Rom J Morphol Embryol 2017;58(1):21-26

6 Gianni L, Pienkowski T, Im YH, et al. Efficacy and safety of neoadjuvant pertuzumab and trastuzumab in women with locally advanced, inflammatory, or early HER2-positive breast cancer (NeoSphere): a randomised multicentre, open-label, phase 2 trial. Lancet Oncol 2012;13(1):25-32

7 Geyer CE, Forster J, Lindquist D, et al. Lapatinib plus capecitabine for HER2-positive advanced breast cancer. N Engl J Med 2006;355(26):2733-2743

8 Verma S, Miles D, Gianni L, et al. EMILIA Study Group. Trastuzumab emtansine for HER2-positive advanced breast cancer. N Engl J Med 2012;367(19):1783-1791

9 von Minckwitz G, Schwedler K, Schmidt $M$, et al. GBG 26/BIG 03-05 study group and participating investigators. Trastuzumab beyond progression: overall survival analysis of 
the GBG 26/BIG 3-05 phase III study in HER2-positive breast cancer. Eur J Cancer 2011;47(15):2273-2281

10 Blackwell KL, Burstein HJ, Storniolo AM, et al. Overall survival benefit with lapatinib in combination with trastuzumab for patients with human epidermal growth factor receptor 2-positive metastatic breast cancer: final results from the EGF104900 Study. J Clin Oncol 2012;30(21):2585-2592

11 Saura C, Oliveira M, Feng YH, et al. NALA Investigators. Neratinib plus capecitabine versus lapatinib plus capecitabine in HER2-positive metastatic breast cancer previously treated with $\geq 2$ HER2-directed regimens: phase III NALA trial. J Clin Oncol 2020;38(27):3138-3149

12 Murthy RK, Loi S, Okines A, et al. Tucatinib, trastuzumab, and capecitabine for HER2-positive metastatic breast cancer. N Engl J Med 2020;382(7):597-609

13 Modi S, Saura C, Yamashita T, et al. DESTINY-Breast01 Investigators. Trastuzumab deruxtecan in previously treated HER2-positive breast cancer. N Engl J Med 2020;382(7):610-621

14 Earl HM, Hiller L, Vallier AL, et al; PERSEPHONE Steering Committee and Trial Investigators. 6 versus 12 months of adjuvant trastuzumab for HER2-positive early breast cancer (PERSEPHONE): 4-year disease-free survival results of a randomised phase 3 non-inferiority trial. Lancet 2019;393(10191) :2599-2612

15 Purmonen TT, Pänkäläinen E, Turunen JH, Asseburg C, Martikainen JA. Short-course adjuvant trastuzumab therapy in early stage breast cancer in Finland: cost-effectiveness and value of information analysis based on the 5-year follow-up results of the FinHer Trial. Acta Oncol 2011;50(3):344-352

16 André F, O'Regan R, Ozguroglu M, et al. Everolimus for women with trastuzumab-resistant, HER2-positive, advanced breast cancer(BOLERO-3): a randomised, double-blind, placebo-controlled phase 3 trial. Lancet Oncol 2014;15(6):580-591

17 Krop IE, Kim SB, González-Martín A, et al. TH3RESA study collaborators. Trastuzumab emtansine versus treatment of physician's choice for pretreated HER2-positive advanced breast cancer (TH3RESA): a randomised, open-label, phase 3 trial. Lancet Oncol 2014;15(7):689-699

18 Johnston SRD, Hegg R, Im SA, et al. Phase III, randomized study of dual human epidermal growth factor receptor 2 (HER2) blockade with lapatinib plus trastuzumab in combination with an aromatase inhibitor in postmenopausal women with HER2-positive, hormone receptor-positive metastatic breast cancer: updated results of ALTERNATIVE. J Clin Oncol 2021;39(1):79-89

19 Tolaney SM, Wardley AM, Zambelli S, et al. Abemaciclib plus trastuzumab with or without fulvestrant versus trastuzumab plus standard-of-care chemotherapy in women with hormone receptor-positive, HER2-positive advanced breast cancer (monarcHER): a randomised, open-label, phase 2 trial. Lancet Oncol 2020;21(6):763-775

20 Chan A, Delaloge S, Holmes FA, et al. ExteNET Study Group. Neratinib after trastuzumab-based adjuvant therapy in patients with HER2-positive breast cancer (ExteNET): a multicentre, randomised, double-blind, placebo-controlled, phase 3 trial. Lancet Oncol 2016;17(3):367-377

21 Kaufman B, Mackey JR, Clemens MR, et al. Trastuzumab plus anastrozole versus anastrozole alone for the treatment of postmenopausal women with human epidermal growth factor receptor 2-positive, hormone receptor-positive metastatic breast cancer: results from the randomized phase III TAnDEM study. J Clin Oncol 2009;27(33):5529-5537

22 Huober J, Fasching P, Paepke S, et al. Letrozole in combination with trastuzumab is superior to letrozole monotherapy as first line treatment in patients with hormone-receptor-positive, HER2-positive metastatic breast cancer (MBC) - results of the eLEcTRA trial. Cancer Res 2009;69(24, suppl) ;doi:10.1158/ 0008-5472.SABCS-09-4094

23 Johnston S, Pippen J Jr, Pivot X, et al. Lapatinib combined with letrozole versus letrozole and placebo as first-line therapy for postmenopausal hormone receptor-positive metastatic breast cancer. J Clin Oncol 2009;27(33):5538-5546

24 Musolino A, Naldi N, Bortesi B, et al. Immunoglobulin $\mathrm{G}$ fragment $\mathrm{C}$ receptor polymorphisms and clinical efficacy of trastuzumab-based therapy in patients with HER-2/neu-positive metastatic breast cancer. J Clin Oncol 2008;26(11):1789-1796

25 Perez EA, Thompson EA, Ballman KV, et al. Genomic analysis reveals that immune function genes are strongly linked to clinical outcome in the North Central Cancer Treatment Group n9831 Adjuvant Trastuzumab Trial. J Clin Oncol 2015;33(7):701-708

26 Hou Y, Nitta H, Wei L, et al. PD-L1 expression and CD8-positive $\mathrm{T}$ cells are associated with favorable survival in HER2-positive invasive breast cancer. Breast J 2018;24(6):911-919

27 Salgado R, Denkert C, Campbell C, et al. Tumor-infiltrating lymphocytes and associations with pathological complete response and event-free survival in HER2-positive early-stage breast cancer treated with lapatinib and trastuzumab: a secondary analysis of the NeoALTTO trial. JAMA Oncol 2015;1(4):448-454

28 Stanton SE, Disis ML. Clinical significance of tumor-infiltrating lymphocytes in breast cancer. J Immunother Cancer 2016;4:59

29 Loi S, Giobbie-Hurder A, Gombos A, et al. International Breast Cancer Study Group and the Breast International Group. Pembrolizumab plus trastuzumab in trastuzumab-resistant, advanced, HER2-positive breast cancer (PANACEA): a single-arm, multicentre, phase $1 \mathrm{~b}-2$ trial. Lancet Oncol 2019;20(3):371-382

30 Emens LA, Esteva F, Beresford M, et al. Results from KATE2, a randomized phase 2 study of atezolizumab (atezo) + trastuzumab emtansine (T-DM1) vs placebo (pbo)+T-DM1 in previously treated HER2+ advanced breast cancer (BC) Cancer Res 2019;79(4, suppl) -PD3-01

31 A randomized, phase ii study comparing trastuzumab and vinorelbine in combination with avelumab or avelumab and utomilumab (41BB/CD137 Agonist), in patients With HER2positive metastatic breast cancer who have progressed on prior trastuzumab and pertuzumab. https://clinicaltrials.gov/ ct2/show/NCT03414658.

32 Jackisch C, Stroyakovskiy D, Pivot X, et al. Subcutaneous vs intravenous trastuzumab for patients with ERBB2-positive early breast cancer: final analysis of the HannaH phase 3 randomized clinical trial. JAMA Oncol 2019;5(5):e190339

33 Zagouri F, Sergentanis TN, Bartsch R, et al. Intrathecal administration of trastuzumab for the treatment of meningeal carcinomatosis in HER2-positive metastatic breast cancer: a systematic review and pooled analysis. Breast Cancer Res Treat 2013;139(1):13-22 